\title{
Linx
}

Revue des linguistes de l'université Paris X Nanterre

$75 \mid 2017$

Imaginaires de la ponctuation. Ordre et inquiétude du discours

\section{Ponctuer en 1550 : l'exemple de Louis Meigret}

\section{Cendrine Pagani-Naudet}

\section{OpenEdition}

Journals

Édition électronique

URL : http://journals.openedition.org/linx/1858

DOI : $10.4000 /$ linx.1858

ISSN : 2118-9692

Éditeur

Presses universitaires de Paris Nanterre

Édition imprimée

Date de publication : 22 décembre 2017

Pagination : 17-34

ISBN : 978-2-84016-308-4

ISSN : 0246-8743

Référence électronique

Cendrine Pagani-Naudet, « Ponctuer en 1550 : l'exemple de Louis Meigret », Linx [En ligne], 75 | 2017, mis en ligne le 23 novembre 2018, consulté le 01 mai 2019. URL : http://journals.openedition.org/

linx/1858; DOI : 10.4000/linx.1858

Ce document a été généré automatiquement le 1 mai 2019.

Département de Sciences du langage, Université Paris Ouest 


\title{
Ponctuer en $1550:$ l'exemple de Louis Meigret
}

\author{
Cendrine Pagani-Naudet
}

1 Plus personne ne conteste aujourd'hui la place qui revient à Louis Meigret dans l'histoire de la grammaire française. Ses positions sur la ponctuation restent toutefois méconnues, au moins dans ce qu'elles ont de plus singulier. Témoin de ce malentendu, le Trésor de la Langue Française qui, à l'article ponctuer, cite Le Tretté de la grammere françoeze ${ }^{1}$ en ces termes : « 1550 punctuer 'mettre la ponctuation dans un texte' (Meigret, Tretté de grammere $\left.: 192^{2}\right) »^{3}$. Le verbe punctuer figure effectivement dans le Tretté de 1550, mais l'interpréter comme un acte graphique, ce que suggère la glose, semble peu compatible avec l'imaginaire linguistique de Meigret, célèbre pour ses positions phonocentristes. La ponctuation, en tant qu'elle participe pleinement au «bâtiment » de langage, n'échappe pas à son attention et à sa verve polémique. Comme en matière d'orthographe, Meigret affirme sa différence, il ne saurait ni être assimilé à ses contemporains, ni être annexé à notre modernité dont il bouscule d'ailleurs nombre de représentations.

\section{La ponctuation, matière nécessaire au « bâtiment de langage »}

Les observations de Meigret sur la ponctuation sont souvent assimilées à celles de Dolet, dont elles se démarquent pourtant de manière radicale. Le rapprochement est un fait de l'histoire éditoriale : le Traité sur la commune écriture ${ }^{4}$ et le célèbre opuscule de Dolet $^{5}$ sur la ponctuation firent en 1545 l'objet d'une réédition commune. L'éditeur prétendait «resjouir l'esprit» du lecteur par cette juxtaposition insolite : la succession des textes souligne en effet non la parenté mais l'incompatibilité de deux imaginaires linguistiques. Incompatibilité que confirme la lecture du Tretté de la grammere françoeze, où Meigret exprime pleinement ses idées la langue et donc sur la ponctuation.

Dolet en 1540 pensait pouvoir aborder la ponctuation de manière isolée ${ }^{6}$. Même si son traité devait prendre place dans un projet linguistique plus vaste, le plan qu'il projetait 
pour son Orateur françois (1540:7) manifeste l'intention d'une étude distincte. La grammaire de Meigret est un ensemble organique, où tout se tient. Aussi ne traite-t-il pas la ponctuation dans un chapitre unique, en marge de la grammaire : une répartition plus diffuse montre les liens inextricables que la ponctuation entretient avec la langue. Pauses, soupirs, entrejets, accents, apostrophe, tous ces sujets Meigret les aborde, essentiellement dans les derniers chapitres, mais aussi «par rencontres». L'ordre de l'exposé indique combien, au-delà de l'inventaire, son approche est irréductible à celle de Dolet. Alors que Dolet exposait une liste de «figures » $(1540: 19)$, mettant sur le même plan tous les signes ${ }^{7}$, Meigret sépare et distingue: les points d'admiration et interrogation d'abord ${ }^{8}$, les points de soupir, de semi-pause, point final et parenthèse ensuite ${ }^{9}$. Interrogation et admiration sont mentionnées dans la continuité du livre IX consacré à la prosodie (id. : 128). Il s'agit en effet d'accents et passé le titre du chapitre, c'est comme tels que Meigret les décrit :

Or avons-nous outre ces trois accents deux autres qui sont d'admiration et d'interrogation: lesquels sont entre eux différents en ce que l'admiration élève la prononciation environ le commencement de la clause, là où l'interrogation le fait communément sur la fin: comme O l'home de bien! Qẹl hom' ẹt' vous? ([1550] 1980 : 137)

$\mathrm{Du}$ reste, interrogation et admiration retiennent peu son attention; dans ce même chapitre, il s'attarde plutôt sur l'apostrophe. La ponctuation, quant à elle, se limite aux points de soupir, de semi-pause, point final et parenthèse. Il faut admettre ici ce que disent les titres des chapitres et leur succession, et que soulignent encore les premiers mots du livre XI : «Reste à expédier la ponctuation (...)» (id.: 139). Pour Meigret, l'admiration et l'interrogation n'appartiennent pas au domaine de la ponctuation.

Dernier chapitre distingué par la mise en page, le livre XI ne clôt pas la grammaire. Deux pages consacrées à la syntaxe suivent en effet, et intègrent la ponctuation à la description de la langue :

Voilà donc quant à ce que [= ce qui] concerne les matières nécessaires pour dresser un bon bâtiment de langage. Il ne reste plus que la partie que les Grecs ont appelé Syntaxis et les Latins constructio, que nous pouvons appeler bâtiment ou construction ou ordonnance bonne de paroles. (id. : 140) $)^{10}$

6 Dans l'édition de 1550 , on passe de la ponctuation à la syntaxe "dans un même paragraphe » (Livet, 1859: 115). Livet a raison de souligner ce point ${ }^{11}$. L'absence de séparation étonne le lecteur moderne mais elle est significative : Meigret en parlant de la ponctuation a surtout parlé de syntaxe. Les signes retenus sont présentés rapidement, encore s'agit-il pour Meigret de substituer aux désignations communément reçues sa propre terminologie. Celle-ci est fondée sur un critère sonore. Entrejet mis à part, les "points" sont désignés selon leur durée de pause: soupir, semi-pause, pause. La progression du propos montre que le problème majeur consiste à déterminer quel signe, de la semi-pause ou du soupir, convient entre deux clauses. Meigret est alors obligé de clarifier ce qu'il entend par clause. Le livre XI juxtapose deux définitions, marquées par une forte implication de l'auteur qui ajuste son propos selon le point de vue considéré. Cohérence globale de l'énoncé ou intégration syntaxique à l'échelle locale :

J'appelle une clause, ou plusieurs ensemble membres d'un propos quant à part soi elle a quelque sentence en soi parfaite, quoiqu'elle semble être prémisse de quelque autre. ([1550] $1980: 139)$

J'appelle clause un bâtiment de langage de nom, ou pronom, avec un verbe, soit que le sens soit parfait ou non : comme lẹs jẹns de biẹn seroẹt lẹ́' plus maleureus de çe monde, s'il n'etoẹt point d'aotre víe qe cẹte çy apręs la mort. (id : 139-140) 
7 Il résulte de cet effort une désagréable impression de dualité, qui complique l'élucidation d'une notion que l'on sent néanmoins décisive. Au demeurant, le mot est déjà familier au lecteur du Tretté: selon une habile stratégie d'imposition du métalangage, Meigret l'a en effet abondamment employé, tantôt pour désigner une unité de construction, tantôt pour évoquer des enchaînements discursifs couplant deux clauses entre elles: clauses de l'interrogeant et clause responsive par exemple (id.: 21). Ces lignes sur la ponctuation sont donc liées par le métalangage au reste de la grammaire.

Meigret a voulu le mot clause, et il lui donne l'épaisseur nécessaire à sa conception de la langue et du discours. Il ne cherche pas à fixer conceptuellement une hiérarchie syntaxique allant de la proposition à la phrase. C'est l'autre mouvement qu'il met en lumière : comment l'énoncé « redistribue sur ses constituants le sens dont il est investi en tant qu'énoncé » (Baratin 1989 : 484). Dès les premières pages de la grammaire, Meigret indique que c'est bien par ce mouvement, du global au local, que s'effectue le découpage des mots :

La diction donc, mot ou vocable, est (comme dit Priscien) la moindre partie entière et entendible d'un langage bâti d'ordre. J'ai dit entier et entendible, à la différence des syllabes d'un vocable polysyllabe : lesquelles, combien qu'entières, ne sont pas toutefois entendibles, et aussi pour celles, lesquelles, combien qu'elles soient entendibles à part soi, ne sont pas dictions entières pour le sens requis suivant le bâtiment de la clause, trait ou propos, comme quand nous disons Pięrr' ęt aojourdhuy a Paris ẹn danjier d'une grand' ęnvíe: ęn d'ęnvíe ne sera pas diction, ni víe aussi, parce que le sens ne le peut souffrir. ([1550] $1980: 20$ )

En dans envie n'est pas un mot, parce que le sens de l'énoncé s'y oppose. Pour lever une ambiguité, Meigret se fonde exclusivement sur le sens et sur le contexte. Le blanc isole visuellement les mots mais il n'est pas "entendible", ce n'est donc pas un signe linguistique pertinent pour le grammairien. Une suite syllabique devient une diction d'après « le sens requis suivant le bâtiment de la clause ». Il en va de même pour la clause. On ne déduit pas de sa structure interne le signe qui en marquera la limite, c'est le sens requis, c'est sa place au sein d'un ensemble plus vaste, le discours, qui déterminera la distribution des soupirs et des pauses. Quelle que soit sa composition (nos critères de subordination ou d'indépendance sont ici inopérants), la clause peut être membre ou partie d'un membre. Le soupir, qui sert « à la distinction des parties d'un membre », peut conjoindre deux clauses, il marque une solidarité syntaxique entre les deux parties d'un membre. Lorsque la clause est un membre elle-même, qu'elle forme une sentence parfaite, mais constitue la prémisse d'une autre, c'est la semi-pause qui convient. Alors que la semi-pause marque une solidarité discursive, le soupir indique un lien plus étroit, et peut être rapproché de la conjonction :

La conjonction est une partie du langage (...) conjoignant les autres espèces des parties : ou les clauses aux clauses avec quelque signification (...) Finalement elle conjoint les clauses: comme il f̧̣t l'home de bien, combien q'il soẹt un grant uzurier.

([1550] $1980: 125-126)$

Cependant, si le soupir « conjoint aussi la clause à la clause » (id. : 139), à la différence de la conjonction, il n'ajoute pas de « signification ». Le « signe » de ponctuation n'a pas chez Meigret de valeur au-delà de sa fonction discriminante et hiérarchisante. Son emploi découle d'un découpage imposé par l'ordonnancement global du discours. Il n'ajoute pas au sens, il le révèle en manifestant les structures de l'édifice ${ }^{12}$. 


\section{La ponctuation ne note pas la prosodie}

11 Pause, semi-pause, soupir, Meigret adopte le vocabulaire des musiciens, accordant ainsi la description de la ponctuation à celle de la prosodie. Les deux domaines en effet sont liés. La première phrase du livre XI précise clairement la nature de ce lien : « Reste à expédier la ponctuation sous le titre des accents : pour autant qu'elle peut les changer » (id. : 139).

Meigret confère à l'intonation une importance capitale dans la maîtrise de la langue française ${ }^{13}$ :

L'accent donc ou ton en prononciation est une loi ou règle certaine pour élever ou abaisser la prononciation d'une chacune syllabe. Et combien que cette doctrine semblera bien nouvelle au pur Français, si est-elle de telle conséquence que si quelqu'un ne les observe, soit par usage ou par doctrine, et qu'il les confonde, l'oreille française s'en mécontentera. (id. : 128)

Pour exposer cette nouvelle "doctrine ", Meigret a recours à un "moyen oculaire » : représenter les sons sur une portée. Meigret se contente de travailler sur deux tons (il ne prétend pas reproduire toutes les inflexions de la voix). Cette notation lui permet d'expliquer la manière dont se règle l'alternance de tons hauts et bas. La mélodie qu'étudie Meigret ne concerne ni les faits d'intonation liés à l'expression des émotions, ni les modulations liées à des effets oratoires. Les énoncés sont uniquement déclaratifs, assez pauvres du point du vue du sens, voire absurdes. La succession des accents est déterminée par la longueur des mots (monosyllabe, dissyllabe, polysyllabe) et par leur enchaînement. Le plan de l'exposé suit cette logique du nombre. Meigret analyse d'abord des enchaînements de monosyllabes, commençant par les cas où « deux monosyllabes se rencontreront au commencement d'une clause", allongent la chaîne jusqu'à neuf monosyllabes. Certains exemples permettent de visualiser le fonctionnement des soupirs et des pauses, transcrits par une barre de mesure :

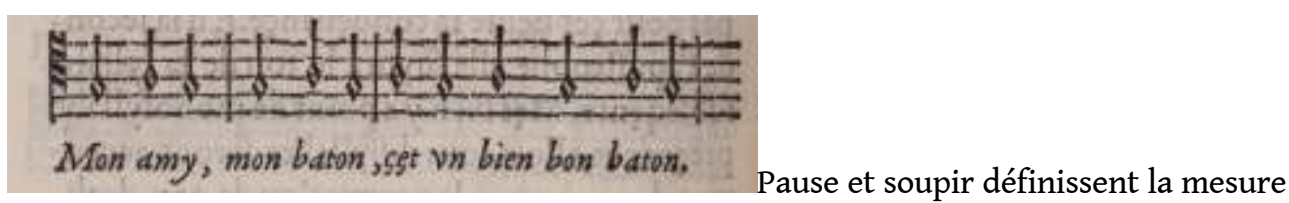

au sein de laquelle se calcule la distribution des accents :

Et notez que toutefois et quantes qu'une pause ou [un] soupir se rencontrera entre les monosyllabes, il faudra juger les premiers selon leur nombre à part: et les seconds selon le leur. (id. : 130)

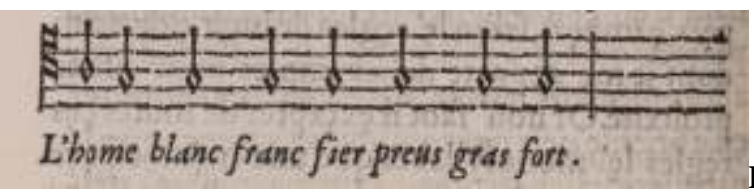

la prosodie soit surdéterminée par le découpage syntaxique. De nombreux exemples ne sont pas construits :

La prosodie est également pensée indépendamment du sens, comme en témoignent les exemples comportant des mots saugrenus - comme: «Lęs megalopolitanes surreparlamęnteront qoẹ qe surreparlamęntassions» (id.: 135) -, inventés pour expérimenter les règles. Un lien existe pourtant, que Meigret indique au début du chapitre consacré à la ponctuation, expédiée «sous le titre des accents : pour autant qu'elle peut les changer ». La présence d'un soupir va en effet avoir une incidence sur le 
nombre dans la mesure où il va permettre d'éviter la chute d'un $e$ muet. On voit dans l'exemple ci-dessous que une, selon sa position, représente une note (une syllabe dans un'ame) ou deux (une fame). De même âme, selon sa position, conserve ses deux syllabes ou du fait de l'élision forme un trisyllabe avec le mot qui suit. Ainsi, l'apostrophe modifie la distribution des accents :

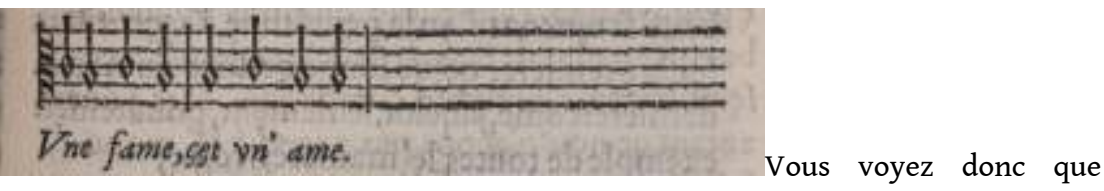

combien que le monosyllabe ç'ȩt précède le dissyllabe une, il n'est pas toutefois aigu, ni la première de une de bas ton : parce qu'en se joignant au subséquent dissyllabe par l'apostrophe, elle forme un trisyllabe, tout ainsi que çe, se joignant à ẹt, fait le monosyllabe ç'ęt. (id: 133)

Ce n'est donc pas un hasard si la question revient à plusieurs reprises dans le Tretté, et si c'est elle qui occupe surtout l'auteur dans le chapitre «Des points d'admiration et interrogation : et de l'apostrophe ». Pour Meigret, l'apostrophe «n'est pas proprement accent, mais elle le fait bien changer » (id. : 137). Et de fait, il élargit considérablement son domaine d'application, s'opposant là encore à Dolet. Ce dernier proposait de noter l'élision au moyen de l'apostrophe uniquement pour les monosyllabes (Dolet, 1540 : 37) ; dans les autres cas, la syllabe non prononcée est écrite. A l'époque du Traité de la commune écriture, Meigret qualifiait déjà «cette restriction aux monosyllabes» de «chatouillement». Il proposait de n'écrire la syllabe que lorsqu'elle est effectivement prononcée et de recourir à l'apostrophe «toutes les fois qu'en la prononciation aucune lettre finale se perd ». En revanche, l'apostrophe :

n'a point de lieu là où il entrevient quelque point autant de virgule que de fin de clause : comme quand nous disons une femme bonne, apprinse, sage, pensera tousjours de son honneur. Autrement tu feras prononciation confuse là où elle requiert être distincte et faite quasi avec quelques pauses. (Meigret, 1542)

16 En 1550, Meigret confirme cette position. Il pratique lui-même l'élision systématiquement, ce que ne peut évidemment pas nous montrer la version modernisée. La solidarité orthographe-ponctuation est rompue par ce choix éditorial. L'apostrophe est en effet un lieu où se manifeste l'unité de la langue. Ainsi l'une des marques de l'interrogation réside dans l'absence d'apostrophe :

quant à il et ęlle, ils ne font point d'apostrophe es verbes précédents par forme d'interrogatoire : comme ȩyme il? ȩyme ȩlle? ([1550] 1980 : 139)

La présence d'un soupir va permettre d'éviter l'apostrophe :

Or faut-il entendre que quant à la copulative $\rho$ [et] et à la disjonctive ou, elles sont au plaisir du prononçant quand ils [= elles] ne conjoignent point clause à clause : de sorte qu'il est en nous de les prononcer sans soupir précédent et lors l'apostrophe y est nécessaire : comme Pięrr' ȩ Ian: ou Pięrr' ou Ian: ou bien avec soupir comme Pięrre, Ł̧ Ian: Pięrre, ou Ian: combien que le premier est plus usité tant entre les autres parties qu'entre les noms: comme lan' ou Iaqelin' ȩym' ou ęn fẹt le sȩmblant. Quoi que ce soit, la bonne oreille y donne ordre. Et si elles couplent les clauses, le soupir y est toujours nécessaire, autrement la prononciation se trouvera de mauvaise grâce: comme si nous disions j'ęyme Dieu de tout mon ceur, ayant ęn luy seul toute ma confianç'ȩ n'ey ęnvíe de la męttr' ęn aotre qe luy: au lieu de ponctuer confiançe. Le semblable de l'ou: comme il faot s'attẹndr' aprȩs çete vi' a une beatitud' etȩrnęlle, ou bien damnaçion pẹrpetuęlle. (id. : 138) $)^{14}$

Voilà l'unique occurrence du verbe ponctuer. Elle apparaît à la faveur d'un développement portant, non pas sur la ponctuation, mais sur l'apostrophe. Dans ce contexte précis, 
"ponctuer confiance » signifie conférer au mot une articulation distincte en préservant la finale de l'élision, ce que permet le soupir. Ce dernier est ici requis parce que la conjonction joint des clauses dont il faut assurer le contour final, ce que permet le soupir en dégageant la dernière syllabe des lois régissant l'apostrophe. L'acte de ponctuer n'est pas un acte graphique ${ }^{15}$, il relève de l'élocution: c'est manifester par l'articulation complète du mot le découpage syntaxique. Cette perspective n'est pas aussi éloignée de la conception ancienne du verbe que ne le suppose M. Stasse :

Ponctuer, sous la forme poncter apparaît pour la première fois chez Christine de Pisan, (...) avec le sens "accentuer en lisant». En 1513, Jean Lemaire de Belges l'utilise pour "marquer les repos dans la récitation d'un poème ». Il faut attendre lui attendre 1550 et le Tretté de la grammere françoeze de Louis Meigret pour trouver le sens de "mettre la ponctuation dans un texte ». Meigret montre l'influence de la ponctuation sur les accents, autrement dit sur l'intonation. (Stasse, 1998 : 179)

La ponctuation que décrit Meigret est celle qui possède une réalisation sonore, qui est "entendible», ce que souligne la référence à la musique. Pour autant, Meigret ne s'enferme pas dans une perspective pneumatique de la ponctuation, la question ne relève pas seulement de la prise de souffle, ou de phénomènes intonatoires au sens large. Respirer, c'est d'abord distinguer. Marquer une pause a une incidence sur l'articulation du mot et sur la syntax ${ }^{16}$. Ce qui importe, c'est l'accès au sens, grâce à une élocution claire, aux silences mesurés.

\section{Ponctuation et ordre du discours}

Ponctuer un texte, c'est donc bien autre chose que «mettre des signes de ponctuation dans un texte ». C'est, en assurant les contours sonores du mot, conjurer toute forme d'équivoque dans l'interprétation du texte, manifester l'ordre du discours et participer à sa stabilité dans les transferts entre l'oral et l'écrit. Les signes de ponctuation contribuent à la restitution fidèle de ce qui a été transcrit: l'écrit n'est en effet que le « dépôt » de l'oral (Meigret, 1548 : 5). Cette idée, Meigret ne cesse de l'affirmer. Préserver l'intégrité de la parole prononcée, garantir l'identité de l'écrit et du dit, voilà les enjeux de la grammaire. Comprendre ce phonocentrisme radical et militant suppose de recomposer le contexte linguistique et juridique de la France de 1550.

Revenons ainsi à la notion de clause. Dans quel esprit Meigret l'emploie-t-il ? Suffira-t-il, pour lui donner du sens, de trouver un équivalent contemporain? En son temps, Meigret est le seul grammairien à en faire un usage régulier ${ }^{17}$. Après lui, peu d'auteurs retiennent le mot: Robert Estienne parce que son Traicté de la grammaire françoise est en grande partie une adaptation du texte de Meigret; Ramus parce qu'il partage bon nombre de ses positions. Bosquet en fait un usage plus vivant, articulant deux plans, ponctuation syntaxique, distinguant les clauses et établissant fermement le sens, et ponctuation pneumatique prenant en compte l'haleine de l'orateur :

Attendu que comme en la poursuite du parler, il est necessaire que nous faisons aucune distinction, ou pose, à celle fin, que celuy qui lit, ou escoute : entende plus facilemen la diuersité des clauses; \& aussy que celuy, quy parle, ou lit reprenne quelquefois son haleine, \& la conforte; pour puis apres prononcer auec plus grande vehemence; Le semblable se doit obseruer en l'escriture, à fin que par certain signe, \& marque soit ostée toute confusion des clauses (lesquelles certainement seroient confuses, sy elles n'estoient distinguées) (Bosquet, 1586 : 137-138) 
En dehors de ces trois auteurs, rares sont ceux qui admettent dans leur métalangage un terme qui semble plutôt relever de la langue juridique ${ }^{18}$. Certes le mot est installé dans la tradition rhétorique (Millet, 1997) et invoquer cette source pourrait suffire. Toutefois, convoquer le domaine du droit permet d'éclairer pertinemment l'œuvre de Meigret et d'expliquer d'autres traits lexicaux et syntaxiques ${ }^{19}$. Hausmann avait déjà souligné la présence de juristes dans le milieu familial de Meigret, influence sensible dans son vocabulaire (1980b : 50-52). D. Kibbee a opportunément «pouss[é] un peu plus loin ces liens entre la pensée grammaticale et la pensée juridique » (2003: 63). Il insiste sur le contexte de rédaction du Tretté, "époque où l'on exige la transformation d'abord d'un droit coutumier en un droit écrit, et ensuite d'un droit écrit en latin à un droit écrit en français » $(2003: 63)$.

Dans le cadre de cette insistance sur la stabilité de la langue pour faciliter l'interprétation du droit coutumier, les decriptions de Meigret servent donc à fixer l'usage et à accorder des usages différents. (2003:69)

Les condamnations que formule Meigret à l'encontre des poètes, praticiens, femmes et mignons efféminés ne seraient «pas tant une réaction contre la variation que contre la fraude, l'abus, l'inconvénient que présente une langue mal réglée » (Kibbee 2003: 70). Dans ce contexte, on conçoit que le rôle assigné à la ponctuation soit d'abord de manifester l'ordre et de stabiliser le dit. Organisatrice du discours, la ponctuation assure la clarté de sa réalisation orale, clarté qui est la condition de sa fidélité. Meigret ne commente pas tant la démarcation graphique que l'incidence sur la perception auditive : ces marques correspondent à des pauses, dont la durée est un renfort à l'articulation logique des clauses. Le choix du vocabulaire musical ne permet pas seulement de rappeler le primat de l'oral, il permet aussi de hiérarchiser les pauses par leur durée, hiérarchie qui n'apparait pas dans le code visuel. Dans sa recherche d'une norme d'intercompréhension, Meigret semble aspirer à un idéal de figement, de pétrification de la matière scripturale, de manière à conjurer tout mouvement, tout glissement, tout tremblement - au sens strict, toute inquiétude - au moment des transferts de l'oral à l'écrit. Le discours est un bâtiment, dont les fondations doivent être solides. C'est pourquoi il importait de rétablir "chacune lettre en sa propre puissance " (Meigret, 1548 : 3). Pour la ponctuation comme pour les lettres, Meigret aspire à l'univocité des signes.

\section{La ponctuation ne transcrit pas les émotions}

Face à cet ordre qui seul préoccupe le grammairien, se dessine un espace d'inquiétude, qu'il peut difficilement ramener à son activité. Les règles ne valent que pour un « langage bâti d'ordre ». Elles n'opèrent pas sur les "plaintes sans forme de paroles ». Le discours dominé par la passion semble être hors du champ de compétence du grammairien : c'est ainsi que peut se comprendre la manière dont Meigret aborde l'affectivité, sans chercher à la rapprocher de la ponctuation ou de la prosodie. Nouveau dépaysement pour le lecteur contemporain, habitué, sous la catégorie confuse de l'exclamation, à glisser d'un domaine à l'autre. Le texte du Tretté offre quant à lui toutes les résistances possibles à de tels amalgames.

Rappelons d'abord les limites que Meigret assigne à la ponctuation. La ponctuation ne note pas l'intonation, laquelle dépend de la structure syllabique des mots et de leur enchaînement. Sans répondre à la question de la prévalence de la syntaxe par rapport à la 
structure prosodique, sans nier les interactions, Meigret du moins distingue les deux domaines. Rappelons aussi que Meigret exclut de la ponctuation l'admiration et l'interrogation : il s'agit d'accents. Dès lors que nous raisonnons sur l'admiration ou sur l'exclamation, nous ne parlons déjà plus de la ponctuation au sens où l'entend Meigret. Rappelons enfin que sur ce " point » d'admiration et son usage, il ne dit à peu près rien. Dolet avait pour sa part été plus précis, établissant explicitement un lien entre point d'admiration, notation des affects, et interjections :

l'admiratif (...) eschet en admiration procedante de joye, ou detestation de vice, \& meschanceté faicte. Il convient aussi en expressionde soubhait, \& desir. Brief : il peult estre par tout, ou il y a interjection. (Dolet, $1540: 23$ )

Meigret choisit d'étudier ces matières séparément : le point d'admiration est un accent, qu'il mentionne au début du livre X, l'interjection est une partie du discours, auquel il consacre le livre VIII. Remarquable chapitre, qui apporte «à la définition un peu sèche de Priscien une description impressionnante de l'affectivité humaine » (Hausmann, 1980b : 197) :

L'interjection est une voix d'une passion excessive: soit par admiration, courroux, joie, mélancolie ou épouvantement. Laquelle nature a inventée d'une par trop grande émotion d'esprit, par colère ou joie: ou bien d'une par trop contrainte retraite à cause d'une grande appréhension mélancolique épouvantable ou admirable: de sorte qu'il n'est pas au pouvoir de l'homme, étant cette passion formée, d'user de quelque modérée façon de parole. Et pour tant voyons-nous que les enflambés de courroux parlent brutement, sans ordre et en paroles syncopées, pour la trop grande élévation d'esprits que la colère enflambée émeut dedans les veines : là où au contraire la passion mélancolique repousse d'une grande détresse, peur ou épouvantement les esprits : de sorte qu'à peine peut la langue former rien promptement que quelque voix plaintive: comme hao, hay. Et si la passion quelquefois continue, nous usons de paroles selon que la véhémence commence quelque peu à décliner : comme helas mon Dieu sera ç'a jamęs qe le monde te hẹrra? ha qe de maleurs ẹn çe monde! ([1550] 1980 : 127)

Bien qu'elle figure dans la liste des parties du discours (id.: 21), Meigret ne dit pas de l'interjection qu'elle est une partie du discours, il dit qu'elle " est une voix ». Précaution significative et nécessaire: l'interjection apparaît précisément lorsque la passion compromet l'élaboration du discours.

ha ha he he: dont quelquefois nous usons si excessivement par une grande dilatation d'esprits que nous n'y pouvons donner suite de quelque bâtiment de paroles pour en rendre la cause. (id. : 127)

Elle est en outre à peine au-dessus des manifestations physiques de la passion. Son caractère naturel et universe ${ }^{20}$ explique que Meigret renonce à en donner la liste complète. Les interjections ne sont pas propres à la langue française dont il dresse la grammaire :

Il en est d'autres dont je me déporte, pour autant que la plupart d'elles tiennent d'une inconstance, impatience ou surprise : attendu que la fragilité de l'homme est prompte à se rendre et tourner à la diversité des passions qui s'offrent. Mais comme ces passions soient communes à toutes nations et que la seule nature les engendre sans aucun discours, il advient qu'elles sont presque toutes unes à tous peuples et langues : tout ainsi que les soupirs et plaintes sans forme de parole. (id. : 128)

Le grammairien peut-il raisonner sur de telles matières? L'émotion contrevient à la bonne élocution dont Meigret a décrit les règles. Dès lors que les affects prennent le dessus, les mots ne sont plus convenablement articulés, il n'est plus possible de régler l'accent sur des "paroles syncopées». Les principes d'accentuation qu'étudie le 
grammairien ne valent qu' « en un langage modéré sans colère ni complainte : car alors chacun les corrompt selon sa manière de cris et plaintes (id.: 139). L'expression des émotions relève de la sphère intime, échappant à la norme collective, sauf lorsque la contention des passions permet de rétablir les conditions d'un «bâtiment de langage ». Réapparaissent alors des énoncés construits, dont on peut déterminer le contour final : ce peut être l'admiration (ha qe de maleurs ẹn çe monde!) ou l'interrogation (helas mon Dieu sera ç'a jamęs qe le monde te hęrra?). La passion "excessive " que manifeste l'interjection n'implique donc pas une intonation spécifique, et l'interjection en emploi isolé n'est accompagnée d'aucun signe conventionnel. Rien dans la grammaire de Meigret ne lie le point d'admiration à l'interjection: dans les rares exemples (6 occurrences dans tout le Tretté) où il apparaît, il n'accompagne jamais l'interjection en emploi isolé, il indique l'intonation d'un énoncé construit.

En somme, bien qu'il décrive longuement les manifestations linguistiques de l'affectivité, Meigret semble en même temps l'écarter du champ de la grammaire. L'expression des affects et les conventions de leur représentation linguistique relèvent, il est vrai, à son époque plutôt de la rhétorique. C'est elle qui prend en charge le travail de modulation de la voix. Mais il est vrai que l'articulation entre les disciplines ménage quelques zones grises et le domaine de l'exclamation en fait partie. Fouquelin rappelle ainsi qu'il revient au grammairien d'enseigner à l'enfant comment prononcer « clairement et apertement ", comment

distinguer les virgulles, les mẽbres, les périodes doucemẽt de son aleine entrecoupee, retenue et reprise: Quand dy-je l'enfant aura apris tout cela du grammairien : Alors le Rhéteur lui montrera de quelle variété et inflexion de vois il faudra user en toutes sentences, figures \& affections de l'oraison. (Fouquelin, 1555 : 113-114)

L'exclamation précisément est une figure ${ }^{21}$. Si le terme même est absent chez les grammairiens, il appartient au vocabulaire de la rhétorique. Fouquelin en propose la définition suivante :

Exclamation est un cri et elevement de vois inventé pour augmenter \& amplifier : Lequel est souventeffois declareé par ces notes ô, helas, las, ah, hé, \& autres semblables., comme en la traduction : ô grande cruauté, ô envie de fortune plus gréve que langue ne sçauroit exprimer \&c. (Fouquelin, 1555 : 104)

Fouquelin caractérise l'exclamation par son intonation, mais il va plus loin en précisant l'effet recherché. Pour illustrer cette brève définition, il cite d'abord une série d'interjections, puis des énoncés plus complexes : aucun point d'admiration n'apparaît dans cette partie du texte. En revanche, ils figurent bien à la fin des vers de Ronsard cités en complément :

O dous parler, dont l'apas doucereus

Nourrit encor la laim de ma memoire!

O front d'amour, le trophee \& la gloire ! (id. : 104)

33 Signe de l'instabilité de l'usage? Le point d'admiration est peu employé dans les imprimés de cette époque. L. Biedermann-Pasques s'interroge sur ces réticences et envisage l'affrontement entre anciens et modernes, «l'emploi du point admiratif étant un signe modernité » (1995: 17). Mais le point d'admiration pose aussi de nombreux problèmes : celui de sa place (à l'intérieur de la phrase ou à la fin ?), et celui de sa valeur : celle-ci n'est pas si clairement définie que celle des autres signes. La réticence de Meigret et de ses contemporains semble tout à fait justifiée par l'inconsistance de ce signe. La description plus détaillée qu'en donne Dolet montre surtout son extrême polyvalence et 
l'impossibilité d'en régler l'usage. Bosquet en cherchant à approfondir cette matière glisse inévitablement vers la rhétorique :

Inventé pour augmenter, \& amplifier, ou flechir la voix d'une harmonie dissemblable, selon l'affection de l'oraison: c'est à dire, de voix apre, eguë, precipitée, interrompuë, lamentable, ou plaisante, \& joyëuse (...) Et signamment il peut avoir lieu, par tout, où il se rencontre, auec aucune interjection: A la fin duquel il convient aussy arrester la voix, \& la hausser d'un ton lamentable, \& interrompu : ou autrement, selon les figures, \& affections de l'oraison. (1586:145)

On en revient au partage des disciplines : l'admiration, son codage par un signe fixe, estelle une question pertinente pour le grammairien? N'est-ce pas à l'orateur de trouver les endroits où l'intonation doit s'élever? D'autre part, dans l'esprit d'un Meigret, soucieux d'établir une norme, de stabiliser les règles de l'intercompréhension, que faire avec un signe dont la marge d'interprétation est indécise, au «contenu plus indiciel qu'informationnel» (Seguin, 2000) ? Meigret s'en tient donc prudemment à sa valeur prosodique, valeur elle-même restreinte à l'élévation de la voix, déterminant le contour final d'une clause. Saluons cette circonspection et un auteur dont la lecture nous permet d'appréhender une étape dans la construction de problèmes qui depuis cette époque suscitent intérêt et perplexité chez les linguistes.

\section{BIBLIOGRAPHIE}

BARATIN, M., 1989, La Naissance de la syntaxe à Rome, Paris, Editions de Minuit.

BIERDERMANN-PASQUES, L., 1995, « Approche d'une histoire du point d'exclamation », Faits de langue, 6, p. 13-22.

BOSQUET, J., 1586, Elemens ou institutions de la langue françoise, Mons, Charles Michel.

CATACH, N., 1994, La Ponctuation, Paris, PUF.

COLOMBAT, B., FOURNIER, J.-M., AYRES-BENETT, W. (éd.), 2011, Grand Corpus des grammaires françaises, des remarques et des traités sur la langue XIV ${ }^{\mathrm{e}}$ XVII ${ }^{\mathrm{e}}$ siècles, Paris, Classiques Garnier Numérique.

DAUVOIS, N., DURRENMATT, J., (ed.), 2011, La ponctuation à la Renaissance, Paris, Classiques Garnier.

DEMONET, M.-L., 2006, « Eh / hé : l'oralité simulée à la renaissance », Langages, 161, L'interjection : jeux et enjeux, Paris, p. 57-72.

DEMONET, M.-L., 2000, « Ponctuation et narration chez Rabelais et ses contemporains », La Licorne, 52, p. 37-62.

DOLET, E., 1540, La Manière de bien traduire une langue en aultre. D'advantage. De la punctuation de la langue Françoyse. Plus. Des accents d'ycelle, Lyon, Etienne Dolet.

ESTIENNE, R., 1557, Traicté de la grammaire françoise, Paris, Robert Estienne.

FOUQUELIN, A., 1555, La Rhétorique françoise, Paris, André Wechel. 
HAUSMANN, F. J., 1980a, Louis Meigret. Le Traité de la Grammaire française (1550). Le Menteur de Lucien- Aux Lecteurs (1548), Tübingen, Narr.

HAUSMANN, F. J., 1980b, Louis Meigret, humaniste et linguiste, Tübingen, Narr.

KIBBEE, D., 2003, « Louis Meigret lyonnais et les politiques de la langue française à la Renaissance ", dans G. Defaux (eds.), Lyon et l'illustration de la langue française à la Renaissance, ENS édition.

LARDON, S., THOMINE, M. CL., 2009, Grammaire du française de la Renaissance, Paris, Classiques Garnier.

LIVET, CH.-L., 1859, La grammaire française et les grammairiens du XVI ${ }^{e}$ siècle, Paris, Didier et Cie, A. Durand.

MEIGRET, L., 1542, Traicté touchant le commun usage de l'escriture françoise, Paris, Denis Janot.

MEIGRET, L., 1545, Traicté touchant le commun usage de l'escriture françoise, Paris, Vve Marnef.

MEIGRET, L., 1548, Le Menteur ou l'incredule de Lucien traduit de Grec en Frãçoe par Louis Meigret Liones , Paris, Chrétien Wechel.

MEIGRET, L., 1550, Tretté de grammere françoeze, Paris, C. Wechel.

MILLET, O., 1997, « Entre grammaire et rhétorique : à propos de la perception de la phrase au XVI e siècle ", L'Information grammaticale, 75, p. 3-9.

PAGANI-NAUDET, C., 2009, « Grammaire et prosodie dans Le Tretté de la grammere francoeze de Louis Meigret, Grammaire et prosodie », Grammaire et Prosodie, D. Roulland (ed.), Presses Universitaires de Rennes.

PAGANI-NAUDET, C., 2013, « L'architecte, figure du grammairien? », Le discours et la langue 4.2, p. 27-37.

PAGANI-NAUDET, C., 2014, « La langue des grammairiens est-elle exemplaire », dans AyresBennett, W. Et Rainsford, T. M. (eds), L'Histoire du français: État des lieux et perspectives, Paris Garnier.

RAULT, J., 2014, « De la pause à la valeur en langue. Grammaticalisation des signes de ponctuation », Congrès Mondial de Linguistique Française 2014.

SEGUIN, J.-P., 2000, « Le point d'exclamation dans Le Père de famille de Diderot : Une ponctuation musicale?», La Licorne, 52, p. 93-111.

STASSE, M., 1998, « Retour aux sources : point final ! », dans Defays J.-M., Rosier L., Tilkin, F. (eds), A qui appartient la ponctuation ?, Bruxelles-Paris, De Boeck, p. 179-190.

\section{NOTES}

1. Le Tretté de la grammere françoeze paraît chez Chrétien Wechel en 1550 dans une graphie entièrement phonétique. Nous citons d'après l'édition modernisée de F. J. Hausmann 1980.

2. C'est à l'édition Foerster (Heilbronn 1888) que fait référence le TLFi.

3. D'après le site du TLFi, http://atilf.atilf.fr/, consulté le 03/04/2015.

4. Meigret, 1545, Traicté touchant le commun usage de l'écriture françoise faict par Loys Meigret, Lyonnois, Paris, Marnef. La première édition date de 1542, Paris, Denis Janot. 
5. Pour la première édition: Dolet, 1540, La manière de bien traduire une langue en aultre. D'advantage. De la punctuation de la langue Françoyse. Plus. Des accents d'ycelle, Lyon, Etienne Dolet. Nous citons Dolet d'après l'édition de 1540.

6. La ponctuation étant pour Dolet universelle - selon lui, les langues «n'ont qu'une ponctuation seulement» (1540:17) -, elle doit effectivement dans son esprit pouvoir être étudiée indépendamment de la grammaire d'une langue nationale.

7. A savoir : point à queue, comma, point rond, point interrogeant, point admiratif, parenthèses. Pour étude détaillée de son système, voir Catach, 1968 et Demonet, 2000.

8. Livre X, « Des points d'admiration et interrogation : et de l'apostrophe » ([1550] 1980 : 137).

9. Livre XI « Des points de soupir, de semi-pause, point final et parenthèse » (id. : 139).

10. L'édition d'Hausmann introduit une rupture (blanc, et changement d'alinéa) qui masque cette continuité du propos.

11. D'une manière générale, Meigret recourt très peu au paragraphe, les articulations sont marquées par un changement de chapitre ou de section. Toutefois, il lui arrive d'y recourir, le passage à la ligne étant en ce cas renforcé par une marque discursive: "Or qant ao' prepoziçions » $\left(1550: 119 \mathrm{r}^{\circ}\right)$, « Venons meintenant » $\left(\right.$ id. : $\left.111 \mathrm{v}^{\circ}\right)$.

12. La métaphore du bâtiment est également importante dans l'imaginaire linguistique de Meigret, voir Pagani-Naudet 2013.

13. A la différence de Dolet, selon qui cet aspect n'est pas pertinent pour le français (Dolet, 1540 : 25).

14. Usage différent de Dolet, selon qui la virgule se met « devant ce mot ou et semblablement devant ce mot et » $(1540: 21)$. Voir Catach, $1994: 31$.

15. Meigret distingue l'acte de ponctuer et le signe qui le marque à l'écrit : « si nous y entrejetons un soupir, que nous dénotons par un point à queue, ou une pause, que nous marquons à deux points ronds ou point final (...).» ([1550] $1980: 138)$

16. Sur le spectre sémantique de pause voir Rault 2014.

17. C'est du moins ce qu'enseigne une requête portant sur l'ensemble des auteurs du Grand corpus des grammaires françaises, des Remarques et des traités de langue: sur 69 occurrences (de clause ou clauze), 39 figurent chez Meigret. Viennent ensuite Bosquet (7 occurrences) et Robert Estienne (6 occurrences). Les autres occurrences correspondent à des emplois très ponctuels, ou figurent dans les exemples.

18. C'est la valeur qui domine dans les différentes occurrences relevées dans le Dictionnarium latinogallicum de Robert Estienne (Paris, Charles Estienne, 1552). Dans le Dictionnaire francois-latin : auquel les mots françois, avec les manieres d'user d'iceulx, sont tournez en latin, c'est le premier sens qui apparaît : «Clause. Il vient de Clausula. La close qui est chasque loy portant peine à l'encontre de ceulx qui feront au contraire ou qui n'obéiront, Sanctio. Mettre en une epistre la derniere close, Epistolam concludere. C'est une clause commune, Caput est translatitium. Clauses entrebrisées par certaines poses, poincts et arrests, Interpunctae clausulae. (J. Dupuys, Paris, G. De Hus, 1573 : 134).

19. Par exemple, l'emploi régulier du subjonctif après comme. Voir Pagani-Naudet (2014).

20. Cette idée figure chez Dubois: "lorsqu'il termine sur l'universalité de beaucoup d'interjections (remarque qui manque chez Donat et Priscien), il semble une fois de plus suivre Dubois qui commence son chapitre par cette idée » (Hausmann 1980b : 197).

21. Sur les rapports entre interjection et exclamation, voir Demonet 2006. 


\section{RÉSUMÉS}

Pour interroger nos représentations sur la ponctuation, nous proposons un retour aux sources, à travers l'un des textes majeurs de la Renaissance : Le Tretté de la grammere françoeze de Louis Meigret. Partisan d'une écriture phonétique, Meigret est particulièrement attentif à la prosodie et aux marques graphiques du découpage du discours et des mots. Il souligne leur complémentarité et leur solidarité avec le reste du discours sur la langue. Ponctuer un texte, c'est en effet bien autre chose que «mettre des signes de ponctuation dans un texte ». C'est, en assurant les contours sonores du mot, conjurer toute forme d'équivoque dans l'interprétation du texte dans le passage de l'écrit à l'oral. Parallèlement pourtant se dessine un espace d'inquiétude : c'est celui du discours dominé par la passion, où les " paroles syncopées » échappent à la juste distribution des accents et au système de ponctuation qui manifeste l'ordre du discours. Bien que la succession des chapitres et le vocabulaire invitent à un rapprochement, Meigret aborde sans les confondre des domaines que l'on aurait désormais tendance à associer spontanément: affectivité et ponctuation.

To question our representations on punctuation, this article suggests to go back to one of the major texts of the Renaissance : LeTretté de la grammere françoeze of Louis Meigret. Supporter of a phonetic writing, Meigret pays particularly attention to the prosody and the graphic marks of the division of the sentence. He underlines their complementarity and their solidarity with the rest of the speech on language. Punctuating a text is more than merely "putting the punctuation marks in a text". Punctuating is, by ensuring the contours of the word, avoiding any form of misinterpretation in the passage from written to oral form. Meigret also studies without confusing them the relations between affectivity (interjections and exclamation) and punctuation.

\section{INDEX}

Mots-clés : ponctuation à valeur expressive, ponctuation à valeur sémantique, diachronie, 16è siècle

\section{AUTEUR}

\section{CENDRINE PAGANI-NAUDET}

UMR 7320, Université Nice Sophia Antipolis 